\title{
All-fiber polarization locked vector soliton laser using carbon nanotubes
}

\author{
C. Mou, ${ }^{*}$ S. Sergeyev, A. Rozhin, and S. Turistyn \\ Photonics Research Group, School of Engineering and Applied Science, Aston University, Birmingham, UK, B4 7ET \\ *Corresponding author: mouc1@aston.ac.uk
}

Received June 13, 2011; accepted August 1, 2011;

posted September 7, 2011 (Doc. ID 149152); published September 23, 2011

\begin{abstract}
We report an all-fiber mode-locked erbium-doped fiber laser (EDFL) employing carbon nanotube (CNT) polymer composite film. By using only standard telecom grade components, without any complex polarization control elements in the laser cavity, we have demonstrated polarization locked vector solitons generation with duration of $\sim 583 \mathrm{fs}$, average power of $\sim 3 \mathrm{~mW}$ (pulse energy of $118 \mathrm{pJ}$ ) at the repetition rate of $\sim 25.7 \mathrm{MHz}$. (C) 2011 Optical Society of America

OCIS codes: $\quad 060.3510,140.4050,250.5530$.
\end{abstract}

Mode-locked ultrafast erbium-doped fiber lasers (EDFLs) have shown many advantages over conventional solid-state light sources in various areas such as optical communication, metrology, sensing, medical application, etc. A range of approaches have been used to achieve mode-locking in EDFLs. The two currently most popular techniques are nonlinear polarization rotation (NPR) [1] and semiconductor saturable absorber mirrors (SESAMS) [2,3], though other methods of mode-locking are actively studied. In practical terms, the search is for an optimal balance between laser performance and cost.

Recently, carbon nanotube (CNT) based saturable absorbers have attracted a great deal of attention due to their inherent nonlinear optical properties, such as fast recovery time, low cost, wide band absorption, ease of fabrication, and low cost $[\underline{4}, \underline{5}]$. So far, various modelocked lasers implemented by CNT have been demonstrated, including fiber lasers [6-11], waveguide lasers [12], and semiconductor lasers [1]3]. Though polarization has been taken into consideration in some works [14-17] when designing or applying CNT in the laser cavity, in general, polarization properties of the CNT mode-locked lasers have not yet been fully explored.

In this Letter, we use a CNT-polyvinyl alcohol (PVA) thin film saturable absorber with absorption peak at $1.5 \mu \mathrm{m}$ region to implement an ultrafast mode-locked EDFL with stable single polarization output. The generated pulses present vector solitons [1-ㅡㄹ 25$]$ in which polarization state is locked and stabilized by nonlinearity. Efficient absorption of CNTs at specific wavelength is determined by the band gap of the specific chiralities of semiconducting single wall CNTs. We use commercially available purified HiPCO single wall CNTs (Unidym) grown through high-pressure $\mathrm{CO}$ conversion. HiPCO CNTs have tube diameter $0.8-1.3 \mathrm{~nm}$, which give a strong optical absorption at the $1000-1600 \mathrm{~nm}$ spectral region. For preparation of the saturable absorber, the $2 \mathrm{mg}$ of CNTs are placed in $10 \mathrm{ml}$ of deionized water containing $10 \mathrm{mg}$ of sodium dodecylbenzene sulfonate (SigmaAldrich) surfactant and then subjected to sonication using commercial ultrasonic processor (Nanoruptor, Diagenode) for one hour at $200 \mathrm{~W}$ and $20 \mathrm{kHz}$. In order to remove residual bundles and impurities, the resulting solution is subjected to ultracentrifugation, 25000 RPM during one hour with Optima Max-XP ultracentrifuge
(Beckman Coulter). We mix the resulting solution with PVA powder and placed in the Petri dish. The CNT-PVA film was then obtained after drying the sample in the desiccator for a few days. The film has a homogeneous distribution of single wall carbon nanotubes on a submicron scale, as confirmed by a featureless surface in optical microscopy. The absorption spectrum of the CNT-PVA saturable absorber shown in Fig. 1 is measured using a Lambda 1050 UV-NIR spectrometer (Perkin Elmer). It has typical features of HiPCO CNTs between 1000 and $1600 \mathrm{~nm}$ with the optical density about 0.4 at $1560 \mathrm{~nm}$.

Figure 2 illustrates the schematic configuration of the CNT mode-locked EDFL. The EDFL constitutes $\sim 2 \mathrm{~m}$ of highly concentrated erbium-doped fiber (EDF Er80-8/ 125 from Liekki) as the gain medium. A fiber pigtailed isolator (OIS) is employed to ensure single direction oscillation of the laser. The laser is pumped via a grating stabilized $980 \mathrm{~nm}$ laser diode (LD) using a $980 \mathrm{~nm} / 1550 \mathrm{~nm}$ wavelength division multiplexing (WDM). A set of commercial diode laser driver and controller is employed to stabilize the performance of the pump.

The nonlinear effects were reduced by minimization of the length between the EDF and the location of a standard fused fiber coupler where $90 \%$ of light is coupled out the laser cavity. An in-line polarization controller was incorporated in the laser cavity for optimization purpose. The involvement of polarization controller does not affect the laser to be self-started. The CNT mode-locker was sandwiched between two standard fiber connector

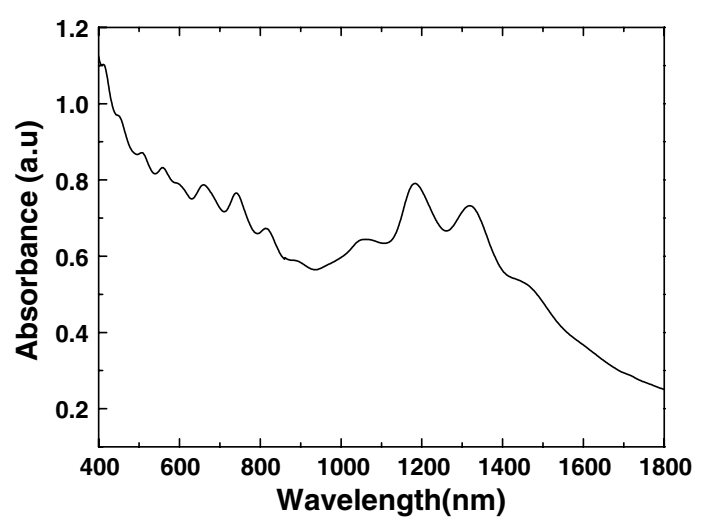

Fig. 1. Absorption spectrum of the CNT-PVA saturable absorber measured by a wideband spectrometer. 


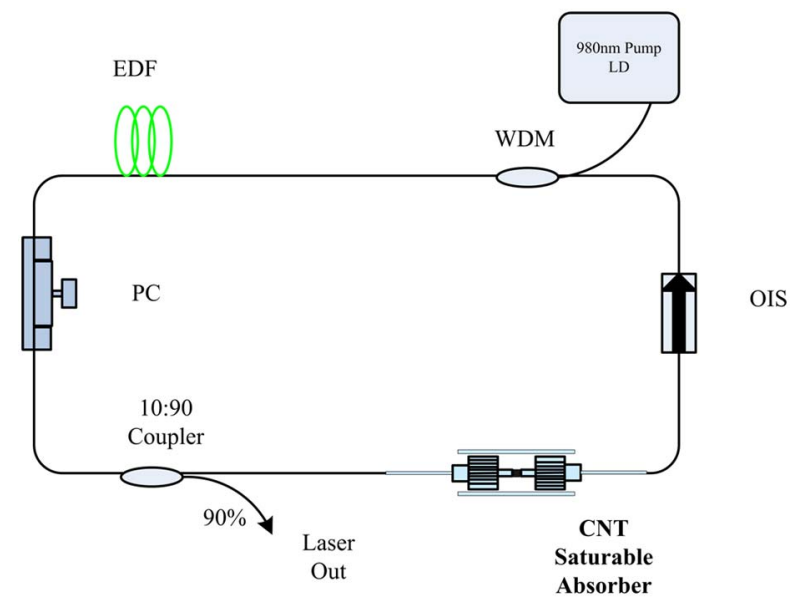

Fig. 2. (Color online) Schematic illustration of the proposed CNT mode-locked EDFL.

ferrules; index matching gel is applied to minimize the transmission loss. This prepackaged saturable absorber is then spliced into the laser cavity. The total length of the laser cavity is $\sim 7.83 \mathrm{~m}$. The whole cavity has an average anomalous dispersion of about $\sim 16.48 \mathrm{ps} / \mathrm{nm} / \mathrm{km}$ that will result in soliton output.

Figure 3 shows a typical output optical spectrum of the EDFL centered at $\sim 1560 \mathrm{~nm}$ with a spectral bandwidth at full width half-maximum (FWHM) of $\sim 3.72 \mathrm{~nm}$. The pronounced Kelly sidebands indicate fundamental soliton shape of the output pulses. A typical pulse train is shown in the inset of Fig. 3 with $\sim 38.9$ ns interval between the two adjacent pulses, thus giving a repetition rate of $\sim 25.7 \mathrm{MHz}$. The output pulses have then been directly fed through a commercial autocorrelator without any preamplification. The measured autocorrelation trace corresponding to the pulse duration of $\sim 583 \mathrm{fs}$ is shown in Fig. 4 .

The calculated time bandwidth product is $\sim 0.26$, which is lower than the theoretical value of 0.315 . This is possibly due to the compression of pulse via the standard fiber from the laser output to the autocorrelator. The EDFL is pumped at $\sim 178 \mathrm{~mW}$ which allows $\sim 3 \mathrm{~mW}$ output power corresponding to energy of $\sim 118 \mathrm{pJ}$.

The high pump power is provided by the high absorption of the gain fiber and high output coupling ratio. The operation stability has been studied by measuring the

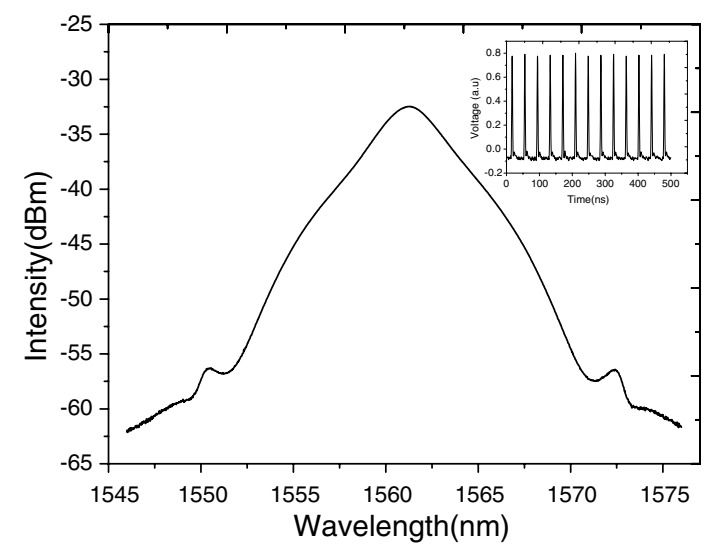

Fig. 3. Output optical spectrum with pronounced Kelly sidebands indicating soliton pulse shape. Inset shows typical pulse train with a repetition rate of $25.7 \mathrm{MHz}$.

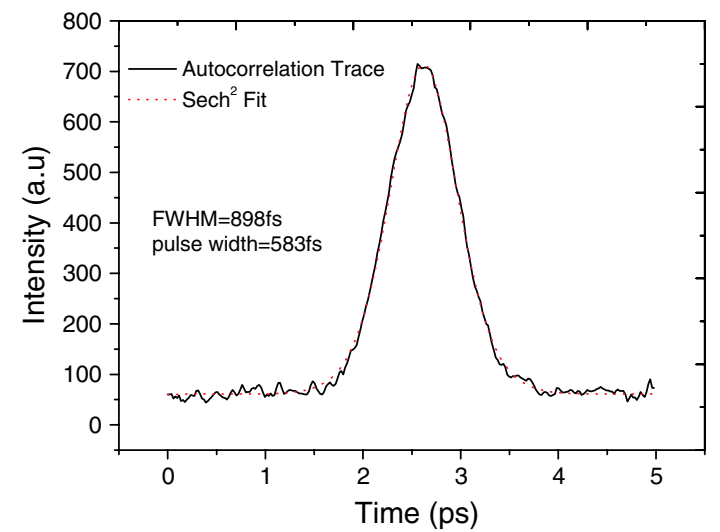

Fig. 4. (Color online) Measured autocorrelation trace of the output pulse showing pulse duration of $\sim 583 \mathrm{fs}$.

radio frequency $(\mathrm{RF})$ spectrum of the laser with an electrical spectrum analyzer. Figure. 5 plots the RF spectrum at the fundamental frequency of laser oscillation showing $60 \mathrm{~dB}$ signal-to-noise ratio with $10 \mathrm{~Hz}$ resolution. This indicates a low relative timing jitter $\Delta t / T$, where $T$ is the round trip time. Applying the approach described in [26] we can estimate $\Delta t / T \approx 6 \times 10^{-5}$, corresponding to a timing jitter $\Delta t \approx 2.3 \mathrm{ps}$.

The polarization properties studied using a commercial polarimeter with $1 \mu \mathrm{s}$ resolution and measurement interval of $1 \mathrm{~ms}$ which corresponds to $40-40000$ round trips are presented in Fig. 6 that shows both the exact polarization state of the output pulse in the Poincare sphere and the evolution of the measured state of elliptic polarization (with the phase-difference of $-\pi / 2$ ) locked by vector soliton and the degree of polarization (DOP) close to $92 \%$.

Figure 6(a) illustrates a stable polarization operation of the EDFL in the Poincare sphere with the axis defined by the three Stokes parameters. Two orthogonally polarized vector soliton states are the eigenstates for this laser and the soliton coupling determine stable slow polarization dynamics. Figure $6(\mathrm{~b})$ presents the measured polarization evolution in terms of optical power and also a stable DOP within the measured time scale. At the laboratory conditions, the output pulses show stable elliptical polarization state for 24 hours without any significant signal degradation. Note that the output polarization state can be controlled by tuning the PC in the laser cavity or after the output coupler.

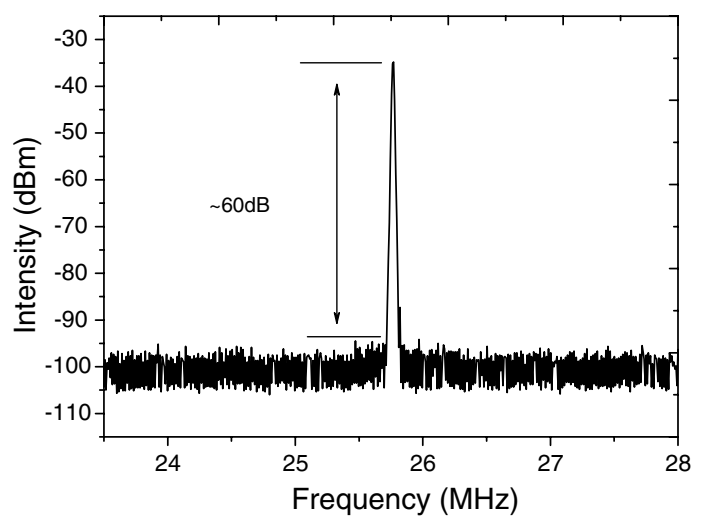

Fig. 5. Measured RF spectrum of the mode-locked EDFL at the fundamental oscillation frequency. 

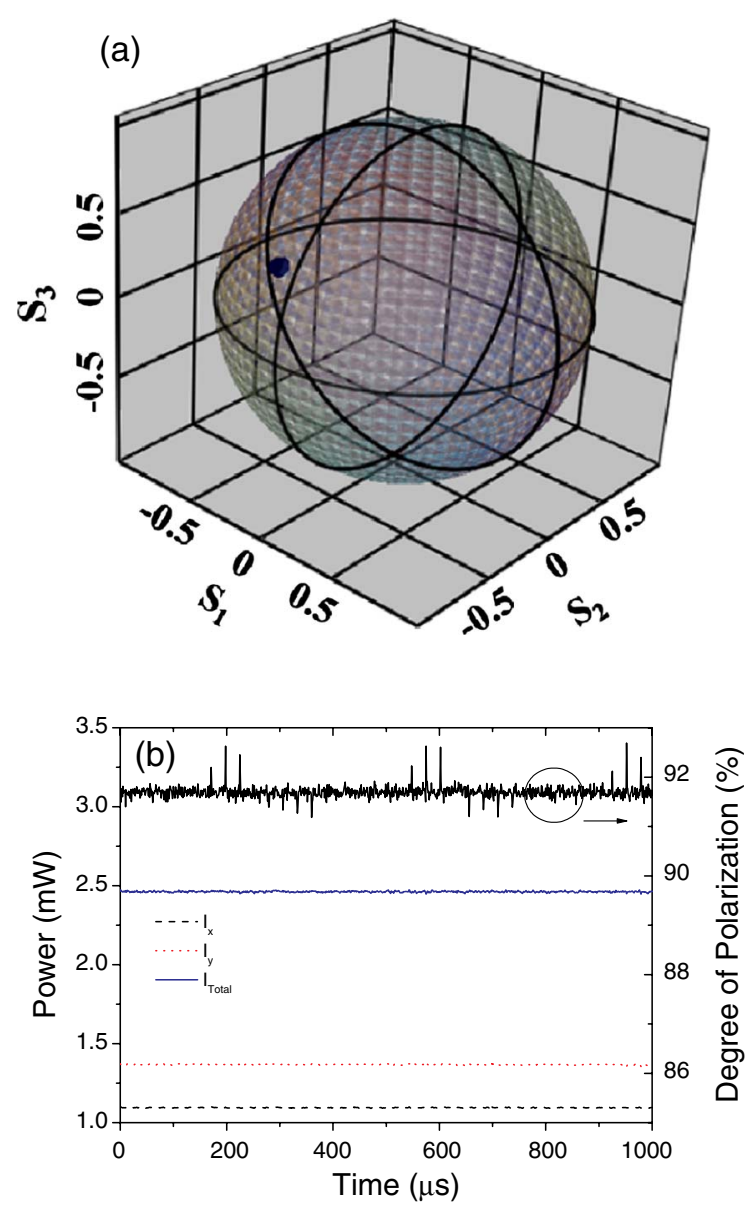

Fig. 6. (Color online) Time evolution of polarization locked vector solitons for the time frame of 40-40000 round trips ( $1 \mu \mathrm{s}-1 \mathrm{~ms})$ in terms of (a) Stokes parameters at Poincare sphere and (b) intensities of orthogonally polarized modes; laser power of $I_{x}$ (black dashed line) and $I_{y}$ (red dotted line); total intensity: $I_{\text {tot }}=I_{x}+I_{y}$ (blue solid line) and degree of polarization (DOP).

In conclusion, we have proposed and demonstrated a CNT-PVA thin film mode-locked EDFL. The laser with relatively simple design generates polarization locked vector soliton pulses with $\sim 583 \mathrm{fs}$ temporal width and an output power of $\sim 3 \mathrm{~mW}$ (pulse energy of $\sim 118 \mathrm{p} J$ ) with a repetition rate of $\sim 25.7 \mathrm{MHz}$ at $1560 \mathrm{~nm}$.

\section{References}

1. K. Tamura, H. A. Haus, and E. P. Ippen, Electron. Lett. 28, 2226 (1992).
2. O. Okhotnikov, A. Grudinin, and M. Pessa, New J. Phys. 6 (2004).

3. M. E. Fermann, A. Galvanauskas, and G. Sucha, Ultrafast Lasers : Technology and Applications (Marcel Dekker, New York, 2003).784 p.

4. S. Y. Set, H. Yaguchi, Y. Tanaka, and M. Jablonski, IEEE J. Sel. Top. Quantum Electron. 10, 137 (2004).

5. S. Y. Set, H. Yaguchi, Y. Tanaka, and M. Jablonski, J. Lightwave Technol. 22, 51 (2004).

6. A. G. Rozhin, Y. Sakakibara, S. Namiki, M. Tokumoto, H. Kataura, and Y. Achiba, Appl. Phys. Lett. 88, 051118 (2006).

7. K. Kieu and M. Mansuripur, Opt. Lett. 32, 2242 (2007).

8. Y. W. Song, S. Yamashita, and S. Maruyama, Appl. Phys. Lett. 92 (2008).

9. Z. Sun, A. G. Rozhin, F. Wang, V. Scardaci, W. I. Milne, I. H. White, F. Hennrich, and A. C. Ferrari, Appl. Phys. Lett. 93 (2008).

10. F. Wang, A. G. Rozhin, V. Scardaci, Z. Sun, F. Hennrich, I. H. White, W. I. Milne, and A. C. Ferrari, Nat. Nanotechnol. 3, 738 (2008).

11. S. Kivisto, T. Hakulinen, A. Kaskela, B. Aitchison, D. P. Brown, A. G. Nasibulin, E. I. Kauppinen, A. Harkonen, and O. G. Okhotnikov, Opt. Express 17, 2358 (2009).

12. G. Della Valle, R. Osellame, G. Galzerano, N. Chiodo, G. Cerullo, P. Laporta, O. Svelto, U. Morgner, A. G. Rozhin, V. Scardaci, and A. C. Ferrari, Appl. Phys. Lett. 89 (2006).

13. Y. W. Song, S. Yamashita, C. S. Goh, and S. Y. Set, Opt. Lett. 32, 430 (2007).

14. Y. W. Song, K. Morimune, S. Y. Set, and S. Yamashita, Appl. Phys. Lett. 90 (2007).

15. Y. W. Song, S. Yamashita, E. Einarsson, and S. Maruyama, Opt. Lett. 32, 1399 (2007).

16. Y. W. Song, S. Yamashita, C. S. Goh, and S. Y. Set, Opt. Lett. 32, 148 (2007).

17. Y. Senoo, N. Nishizawa, Y. Sakakibara, K. Sumimura, E. Itoga, H. Kataura, and K. Itoh, Opt. Express 17, 20233 (2009).

18. D. N. Christodoulides and R. I. Joseph, Opt. Lett. 13, 53 (1988).

19. C. R. Menyuk, J. Opt. Soc. Am. B 5, 392 (1988).

20. V. K. Mesentsev and S. K. Turitsyn, Opt. Lett. 17, 1497 (1992).

21. Y. S. Kivshar and S. K. Turitsyn, Opt. Lett. 18, 337 (1993).

22. S. T. Cundiff, B. C. Collings, N. N. Akhmediev, J. M. Soto-Crespo, K. Bergman, and W. H. Knox, Phys. Rev. Lett. 82, 3988 (1999).

23. B. C. Collings, S. T. Cundiff, N. N. Akhmediev, J. M. Soto-Crespo, K. Bergman, and W. H. Knox, J. Opt. Soc. Am. B 17, 354 (2000).

24. H. Zhang, D. Y. Tang, L. M. Zhao, Q. L. Bao, and K. P. Loh, Opt. Commun. 283, 3334 (2010).

25. H. Zhang, D. Y. Tang, L. M. Zhao, and X. A. Wu, Opt. Express 19, 3525 (2011).

26. D. Vonderlinde, Appl. Phys. B 39, 201 (1986). 